\title{
Correction to: Conditional bias-penalized Kalman filter for improved estimation and prediction of extremes
}

\author{
Dong-Jun Seo ${ }^{1} \cdot$ Miah Mohammad Saifuddin ${ }^{1} \cdot$ Haksu Lee $^{2}$
}

Published online: 22 November 2018

(C) Springer-Verlag GmbH Germany, part of Springer Nature 2018

\section{Correction to: Stoch Environ Res Risk Assess (2018) 32:183-201 https://doi.org/10.1007/s00477-017-1442-8}

In the original publication, Eqs. (3) and (4) should read:

$\widehat{H}^{T}=H^{T}+\alpha C^{T}$

$\Lambda=R+\alpha(1-\alpha) C \Psi_{X X} C^{T}-\alpha H \Psi_{X X} C^{T}-\alpha C \Psi_{X X} H^{T}$

In the above, the $(m \times n)$ conditional bias $(\mathrm{CB})$ gain matrix for the observation vector, $C_{1, k}^{T}$, in $C^{T}=$ $\left[C_{1, k}^{T} C_{2, k}^{T}\right], \quad C_{2, k}^{T}=0$, is given by:

$C_{1, k}=\left[\left(H_{k} \Psi_{X X} H_{k}^{T}+R_{k}\right) G_{1, k}+H_{k} \Psi_{X X} G_{2, k}\right] L_{k}^{-1}$

where

$$
\begin{aligned}
G_{2, k}^{T}= & \left(H_{k}^{T} H_{k}+I\right)^{-1} \\
G_{1, k}^{T}= & G_{2, k}^{T} H_{k}^{T} \\
L_{k}= & G_{2, k}^{T}\left[H_{k}^{T}\left(H_{k} \Psi_{X X} H_{k}^{T}+2 R_{k}\right) H_{k}+H_{k}^{T} H_{k} \Psi_{X X}\right. \\
& \left.+\Psi_{X X} H_{k}^{T} H_{k}+\Psi_{X X}+2 \Sigma_{k \mid k-1}\right] G_{2, k}
\end{aligned}
$$

With the above, Eqs. (5), (7) and (8) in the original publication do not apply. In the original publication, Eqs. (10-12) should read:

$\Lambda_{21, k}=-\alpha \Psi_{X X} C_{1, k}^{T}$

The original article can be found online at https:// doi.org/10.1007/s00477-017-1442-8.

Dong-Jun Seo

djseo@uta.edu

1 Department of Civil Engineering, The University of Texas at Arlington, Box 19308, 416 Yates St. Suite 425, Arlington, TX 76019, USA

2 Len Technologies, Oak Hill, VA 20171, USA
$\Lambda_{11, k}=R_{k}+\alpha(1-\alpha) C_{1, k} \Psi_{X X} C_{1, k}^{T}+\Lambda_{12, k} H_{k}^{T}+H_{k} \Lambda_{21, k}$

$\Lambda_{22, k}=\Sigma_{k \mid k-1}$

In the original publication, Eqs. (19) and (20) should read:

$$
\begin{aligned}
\Sigma_{k \mid k}= & {\left[\varpi_{1, k} H_{k}+\varpi_{2, k}\right]^{-1}\left(\varpi_{1, k} R_{k} \varpi_{1, k}^{T}+\varpi_{2, k} \Sigma_{k \mid k-1} \varpi_{2, k}^{T}\right) } \\
& {\left[\varpi_{1, k} H_{k}+\varpi_{2, k}\right]^{-1} }
\end{aligned}
$$

$K_{k}=\left[\varpi_{1, k} H_{k}+\varpi_{2, k}\right]^{-1} \varpi_{1, k}$

where

$\varpi_{1, k}=\widehat{H}_{1, k}^{T} \Gamma_{11, k}+\Gamma_{21, k}$

$\varpi_{2, k}=\widehat{H}_{1, k}^{T} \Gamma_{12, k}+\Gamma_{22, k}$

In the above, the $\Gamma$ matrices are as defined in the original publication.

The above correction results from Bayesian estimation of $E\left[\left(Z-H M_{X}\right) \mid X\right]$ [see Eq. (36) of the original publication] for which the linear observation equation is given by $\mathrm{X}=\mathrm{G}^{\mathrm{T}} \mathrm{Z}-\mathrm{G}^{\mathrm{T}} \mathrm{V}$ where $G^{T}=\left(U^{T} H\right)^{-1} U^{T}$ with $\mathrm{U}^{\mathrm{T}}$ being some $(\mathrm{m} \times(\mathrm{n}+\mathrm{m}))$ nonzero matrix.

Acknowledgements This material is based upon work supported in part by the National Science Foundation under Grant CyberSEES1442735 and by the National Oceanic and Atmospheric Administration's Joint Technology Transfer Initiative Program under Grants NA16OAR4590232, NA17OAR4590174 and NA17OAR4590184. These supports are gratefully acknowledged.

\section{Appendix 1}

In the original publication, Eqs. (37), (38), (39) and (45) should read: 
$\Sigma_{C B}=\Psi_{X X}-W C \Psi_{X X}-\Psi_{X X} C^{T} W^{T}+W C \Psi_{X X} C^{T} W^{T}$

$\Sigma=(1+\alpha) \Psi_{X X}-W(H+\alpha C) \Psi_{X X}$

$-\Psi_{X X}\left(H^{T}+\alpha C^{T}\right) W^{T}$

$+W\left(H \Psi_{X X} H^{T}+R+\alpha C \Psi_{X X} C^{T}\right) W^{T}$

$\mathrm{W}=\Psi_{X X} \widehat{H}^{T}\left[\widehat{H} \Psi_{X X} \widehat{H}^{T}+\Lambda\right]^{-1}$

$\Delta=\alpha \Psi_{X X} \widehat{H}^{T}\left[\widehat{H} \Psi_{X X} \widehat{H}^{T}+\Lambda\right]^{-1} C M_{X}$

where $\widehat{H}^{T}, \mathrm{C}$ and $\Lambda$ are described in Eqs. (3) and (4).

\section{Appendix 2}

In the original publication, Eqs. (52) through (57) should read:

$$
\begin{aligned}
& \widehat{H}_{1}^{T}=H_{k}^{T}+\alpha C_{1, k}^{T} \\
& \widehat{H}_{2}^{T}=I+\alpha C_{2, k}^{T}=I \\
& \Lambda_{11}=R_{k}+\alpha(1-\alpha) C_{1, k} \Psi_{X X} C_{1, k}^{T}+\Lambda_{12, k} H_{k}^{T}+H_{k} \Lambda_{21, k} \\
& \Lambda_{12}=-\alpha C_{1, k} \Psi_{X X} \\
& \Lambda_{21}=-\alpha \Psi_{X X} C_{1, k}^{T}
\end{aligned}
$$

$$
\Lambda_{22}=\Sigma_{k \mid k-1}
$$

In the original publication, Eq. (58) should be ignored, and Eqs. (59), (60) and (63) should read:

$\varpi_{1, k}=\widehat{H}_{1, k}^{T} \Gamma_{11}+\Gamma_{21}$

$\varpi_{2, k}=\widehat{H}_{1, k}^{T} \Gamma_{12}+\Gamma_{22}$

$\widehat{H}^{T} \Lambda^{-1} H=\varpi_{1} H_{k}+\varpi_{2}$

In the original publication, Eqs. (64) and (65) should be ignored, and the apparent updated error covariance $\Sigma_{a, k \mid k}$ in Eq. (66), which reflects both error covariance and expectation of Type II CB squared, and the scaling matrix B in Eq. (67) should read:

$\Sigma_{a, k \mid k}=B\left[\widehat{H}^{T} \Lambda^{-1} H\right]^{-1}=\alpha \Sigma_{k \mid k-1}+\left[\varpi_{1, k} H_{k}+\varpi_{2, k}\right]^{-1}$

$B=\alpha \Sigma_{k \mid k-1} \widehat{H}^{T} \Lambda^{-1} \widehat{H}+I$

In the original publication, Eq. (68) should be ignored, and Eqs. (69) and (71) should read:

$\widehat{X}_{k \mid k}=\left[\varpi_{1, k} H_{k}+\varpi_{2, k}\right]^{-1}\left[\varpi_{1, k} Z_{k}+\varpi_{2, k} \widehat{X}_{k \mid k-1}\right]$

$K_{k}=\left[\varpi_{1, k} H_{k}+\varpi_{2, k}\right]^{-1} \varpi_{1, k}$ 